\title{
DIMENSIONAMENTO DE CANAIS DE SOFT HANDOFF EM SISTEMAS CDMA
}

\author{
Omar Carvalho Branquinho e Michel Daoud Yacoub
}

\begin{abstract}
Resumo - O planejamento de sistemas celulares CDMA possui características distintas daquelas utilizadas em sistemas que fazem uso de outras técnicas de acesso. As metodologias disponíveis atualmente para o planejamento ou são muito complexas e com considerações que se contrastam, com um nível de sofisticação que não condiz com o grau do empirismo dos parâmetros, ou são muito simples mas pouco rigorosas. Este artigo tem por objetivo a proposta de um metodologia, simples e de fácil aplicação, de dimensionamento de canais de soft handoff que leve em conta tanto a questão do bloqueio de soft handoff quanto a da influência destes canais na interferência .
\end{abstract}

Abstract - CDMA systems exhibit peculiarities that render system planning a task quite distinct from that used in other access technologies. The planning methodologies used today are either complex, using parameters that are not realistic, or simplistic, yielding poor results. This article presents a simple and easy-to-use planning method for determining the number of soft handoff channels. This planning method considers soft handoff blocking and system interference as input parameters.

Palavras-chave: Sistemas CDMA, planejamento celular, soft handoff.

\section{INTRODUÇÃO}

O planejamento de sistemas celulares CDMA possui características distintas daquelas utilizadas em sistemas que fazem uso de outras técnicas de acesso. Particularmente, a técnica de soft handoff exige especial atenção por fazer uso de mais recursos que um mínimo necessário, devendo-se levar em consideração a reserva de canais para a sua implementação. As metodologias disponíveis atualmente para o planejamento de sistemas CDMA ou são muito complexas, e com considerações que se contrastam com um nível de sofisticação que não condiz com o grau do empirismo dos parâmetros, ou são muito simples, e pouco rigorosas. Neste artigo é apresentada uma proposta de metodologia, simples e de fácil aplicação, para o dimensionamento de canais de soft handoff. Esta proposta leva em conta o bloqueio de soft handoff e também considera a interferência causada no processo.

O trabalho inicia-se com uma análise da probabilidade de canais ativos nas regiões de sobreposição para duas e três células. A seguir é apresentado o método para

Omar Carvalho Branquinho está com a Fundação Centro de Pesquisa - CPqD e Michel Daoud Yacoub está com a Universidade Estadual de Campinas - UNICAMP. End. Eletrônicos: omar@cpqd.com.br, michel@decom.fee.unicamp.br dimensionamento de canais de soft handoff utilizando 2 células [1]. Esta estrutura básica permite caracterizar o problema de forma simples. O método é então estendido para 3 células permitindo uma generalização da metodologia proposta para duas células [2]. Um exemplo de aplicação é delineado neste trabalho.

O trabalho está organizado como se segue A seção 2 apresenta as técnicas de planejamento em uso. A seção 3 apresenta a metodologia proposta pelo trabalho. A seção 4 faz análise da probabilidade de canais ativos nas regiōes de sobreposição para duas e três células. A seção 5 trata da probabilidade de bloqueio de soft handoff, que forma a base para a metodologia proposta. A seção 6 apresenta a probabilidade de outage que se relaciona com a interferência. Na seção 7 são apresentadas as estratégias de alocação de canais, utilizadas para os resultados da seção 8 . $\mathrm{Na}$ seção 9 é aplicada a metodologia utilizando os resultados obtidos na seção 8 . Na seção 10 é apresentada a conclusão do trabalho

\section{METODOLOGIAS DE PLANEJAMENTO}

Um usuário móvel na fronteira entre células poderá ser servido por duas ou mais células. Nos sistemas FDMA e TDMA, este fenômeno determina a célula da qual o canal de comunicação será utilizado no cruzamento entre fronteiras, liberando-se assim o canal da célula de origem. Pode-se dizer que, em média, para cada canal capturado na outra célula libera-se um canal na célula de origem. Desta forma trabalha-se com a situação de equilíbrio para se dizer que o handoff nestas tecnologias pouco influi no dimensionamento do sistema. Já no sistema CDMA, um usuário móvel na periferia da célula contribui com uma grande parcela de interferência na outra célula, o que proporciona uma diminuição da capacidade como um todo. O fato de este móvel poder se comunicar com mais de uma célula pode ser utilizado em benefício do sistema, diminuindo-se a potência do móvel e combinando-se os sinais neste móvel para se obter um nível adequado de recepção. Neste sentido. mais do que uma prerrogativa ou uma regalia dos sistemas CDMA, o soft handoff é uma necessidade. Em teoria. o soft handoff não influencia no cálculo da capacidade do enlace reverso, já que os móveis nesta condição transmitem estando ou não em soft handoff. Já o mesmo não se pode dizer com relação ao enlace direto. Neste caso, como um móvel poderá ocupar canais tanto na célula de origem quanto nas demais com as quais a comunicação é adequada, um número adicional de canais deverá ser provido para acomodar esta condição.

Algumas metodologias foram propostas para o dimensionamento do sistema CDMA. Uma mais complexa 
diz respeito à capacidade de Erlang [3]. Em particular, nesta metodologia as considerações se contrastam, com um nível de sofisticação na obtenção das fórmula que não condiz com o grau do empirismo dos parâmetros utilizados nelas. Considera-se ainda uma aproximação Gaussiana em uma somatória em que o próprio limite desta somatória é uma variável aleatória, o que é contestável. Além disto, as fórmulas são deduzidas para a condição de tráfego uniforme, situação pouco encontrada na prática. A dificuldade de manipulação destas fórmulas torna inviável a sua aplicação no dimensionamento de sistemas práticos.

De fato, na prática uma outra metodologia é utilizada [4]. Nesta metodologia, introduzem-se os conceitos de tráfego aparente e tráfego real. O tráfego aparente é aquele pelo qual o sistema pode cobrar do usuário. O tráfego real é aquele efetivamente tratado pelo sistema. Um usuário na condição de soft handoff utiliza mais recursos do que um mínimo necessário. Por outro lado, ele não poderá pagar por se encontrar na condição de soft handoff. Para o dimensionamento de canais para satisfazer a condição de soft handoff, utiliza-se o fator de carga dado pela razão entre a carga real e a carga aparente. A carga real é então determinada multiplicando-se o fator de carga pela carga aparente [1,2].

A carga aparente é obtida pela fórmula de Erlang, para um dado grau de serviço e um número de canais para o qual o nivel de interferência encontra-se em patamares aceitáveis.

Tanto nesta metodologia quanto nas anteriores, nada se diz a respeito do bloqueio de soft handoff. Note ainda, nesta última metodologia, que nada se conjectura a respeito da interferência causada pela utilização dos canais de soft handoff. Por outro lado, este esquema é extremamente simples de ser utilizado, o que o torna muito popular em planejamentos práticos.

\section{METODOLOGIA DE DIMENSIONAMENTO DE CANAIS DE SOFT HANDOFF}

Em comun com a metodologia mais simples e descrita no item anterior. o método proposto faz uso do tráfego aparente para se determinar o tráfego real. Em comum com a metodologia mais complexa, o método proposto se preocupa com a questão da interferência. Em adição a ambas leva-se em consideração o bloqueio de soft handoff. A determinação do tráfego real na metodologia proposta, por outro lado, será consequiência tanto do bloqueio de soft handoff quanto da interferência.

Dado um número nominal para o qual o nível de interferência encontra-se em patamares aceitáveis, determina-se o tráfego aparente através da fórmula de Erlang em função do grau de serviço especificado. Para um fator de sobreposição entre células, adicionam-se ou subtraem-se canais para atender aos requisitos de probabilidade de bloqueio de soft handoff e de interferência. Note que apenas acrescentando-se canais aos canais nominais diminui-se a probabilidade de bloqueio de soft handoff mas, por outro lado, aumenta-se a probabilidade de interferência. No outro extremo, apenas subtraindo-se canais dos canais nominais a interferência não ultrapassará aquela obtida com o uso de canais nominais, mas a capacidade final do sistema diminuirá para se atingir a probabilidade de bloqueio de soft handoff desejada. Um compromisso entre se adicionar e se subtrair uma quantidade adequada de canais em torno dos canais nominais poderá ser atingida para se atender os critérios de projeto de sistema no que diz respeito à questão da interferência e do bloqueio de soft handoff.

O modelo proposto se presta a este objetivo, é de fácil utilização e se aplica a qualquer distribuição de tráfego.

\section{CANAIS ATIVOS NA REGIÃO DE SOBREPOSIÇÃO}

Como ponto de partida para o método proposto é necessário determinar a probabilidade de canais ativos nas regiões de sobreposição. A determinação desta probabilidade será feita primeiramente para o caso de duas células e em seguida expandida para três células.

Para duas células existe somente uma região de sobreposição [1]. A Figura 1 mostra esta condição, onde $\gamma$ é a proporção da área de sobreposição entre duas células.

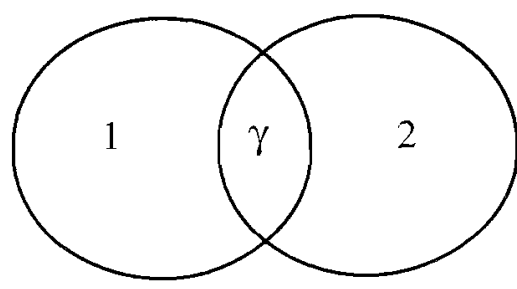

Figura 1. Sobreposição entre duas células.

Neste arranjo trabalha-se com a condição onde existe a geração de tráfego na região de sobreposição e este tráfego deve ser cursado por uma célula alvo, que representa a célula que irá cursar o tráfego originado pela outra célula no processo de soft handoff.

Seja $\boldsymbol{n}$ o número de canais ativos em uma dada célula. A probabilidade $p_{k \mid n}$ de $k$ canais estarem ativos na região de sobreposição entre células, $\boldsymbol{0} \leq \boldsymbol{k} \leq \boldsymbol{n}$, dado $\boldsymbol{n}$, canais ativos é

$$
p_{k \mid n}=\left(\begin{array}{l}
n \\
k
\end{array}\right) \gamma^{k}(1-\gamma)^{n-k}
$$

Supondo $N$ canais na célula. a probabilidade incondicional $p_{k}$ é, portanto, dada por

$$
p_{k}=\sum_{n=k}^{N} p_{k n} p_{n}
$$

onde $p_{n}$ é a distribuição dos canais ativos na célula.

Note que $p_{k}$ é a probabilidade de se terem $\boldsymbol{k}$ assinantes na condição de soft handofft. Adotando-se as premissas usuais de 1) chegada Poissoniana e 2) tempo de retenção de 
chamada distribuído de acordo com uma exponencial negativa, a distribuição $p_{n}$ é dada pela distribuição de Erlang. A distribuição de Bernoulli. como uma aproximação, também será utilizada para este fim.

O arranjo de duas células é limitado para aplicações nráticas, sendo mais apropriado o arranjo de três células. Os resultados para três células são obtidos generalizando-se os resultados obtidos para duas células.

Para três células existem regiões de sobreposição entre duas e três células [2]. A Figura 2 mostra esta condição, $\gamma$ é a proporção da área de sobreposição entre duas ou mais células e $\delta$ é a proporção da área de sobreposição entre três ou mais células.

Para este arranjo trabalha-se com a condição onde existe a geração de tráfego nas regiões de sobreposição e este tráfego deve ser cursado por uma célula alvo. Portanto. em relação à célula alvo as outras duas células apresentam regiões de sobreposição entre duas e três células. Deve-se determinar a probabilidade de canais ativos nestas regiões considerando-se as proporções mostradas na Figura 2.

A análise é feita considerando-se uma célula com área normalizada 1 , ou seja:

$$
1-\gamma+\frac{\gamma-\delta}{2}+\frac{\gamma-\delta}{2}+\delta=1
$$

A região de sobreposição de uma célula com duas células tem uma área proporcional a:

$$
\gamma-\delta
$$

A região de acesso a três células é proporcional a $\delta$. Esta regiāo pode ser aproximada à região de duas células pela seguinte expressão [5.6]:

$$
\delta \cong \gamma^{2}
$$

Esta aproximação permite utilizar somente $\gamma$ como variável de proporção de área para o cálculo das probabilidades envolvidas.

Para três células é necessário utilizar a generalização de Bernoulli [7]. Na generalização de Bernoulli tem-se um conjunto de $r$ eventos. onde cada evento ocorrerá $\left\{k_{1}, k_{2}, \ldots\right.$, $\left.\mathrm{k}_{\mathrm{r}}\right\}$ vezes com suas respectivas probabilidades $\mathrm{p}_{1}, \mathrm{p}_{2}, \ldots, \mathrm{p}_{\mathrm{r}}$, onde

$$
p_{1}+p_{2}+\ldots \ldots \ldots \ldots \ldots+p_{r}=1
$$

$\mathrm{e}$

$$
k_{1}+k_{2}+\ldots \ldots \ldots \ldots \ldots+k_{r}=n
$$

A fórmula generalizada de Bernoulli é dada por:

$$
p_{n}\left(k_{l}, k_{2}, \ldots \ldots, k_{r}\right)=\frac{n_{l}^{\prime}}{k_{l} ! k_{2} ! \ldots \ldots k_{r} !} p_{1}^{k_{l}} p_{2}^{k_{2}} \ldots \ldots p_{r}^{k_{r}}
$$

$\mathrm{Na}$ generalização de Bernoulli, são consideradas as condições para diversas ocorrências de eventos. Para três

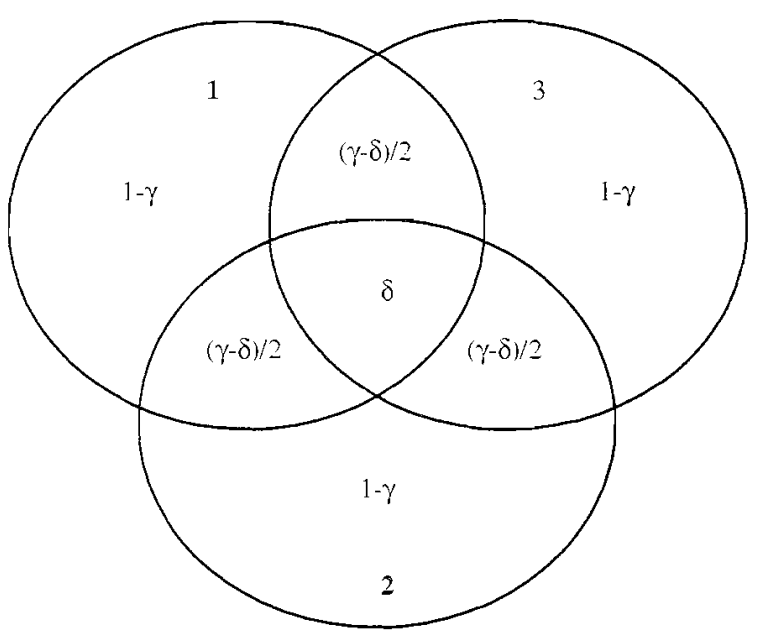

Figura 2. Sobreposição entre três células.

células apenas $k_{1}$ e $k_{2}$, que são as condições de análise para as duas regiões de sobreposição de acesso a duas e três células. Portanto, a probabilidade de $k_{1}$ e $k_{2}$ canais ativos dados $n$ canais ativos é

$$
p_{k_{1} k_{2}=n}=\frac{n ! p_{1}^{k_{1}} p_{2}{ }^{k_{2}}\left(1-p_{1}-p_{2}\right)^{n-k_{1}-k_{2}}}{k_{1} ! k_{2} !\left(n-k_{1}-k_{2}\right) !}
$$

Utilizando (9) e (5) tem-se

$$
\begin{aligned}
p_{k_{1}, k_{2} ! n} & =\frac{n !}{k_{1} ! k_{2} !\left(n-k_{1}-k_{2}\right) !}\left(\frac{\gamma-\gamma^{2}}{2}\right)^{k_{1}} \\
& \times \gamma^{2 k_{2}}\left(\frac{2-\gamma-\gamma^{2}}{2}\right)^{n-k_{1}-k_{2}}
\end{aligned}
$$

Supondo $N$ canais na célula, a probabilidade incondicional $p_{k_{1}, k_{2}}$ é, portanto, dada por

$$
p_{k_{1} \cdot k_{2}}=\sum_{n=k_{1}+k_{2}}^{N} p_{k_{1} \cdot k_{2} l_{i}} p_{n}
$$

Para três células $p_{k_{1}, k_{1}}$ é a probabilidade de existirem $k_{1}$ assinantes na condição de soft handoff na região de sobreposição entre duas células e $k_{2}$ assinantes na condição de soft handoff na região de sobreposição entre três células.

\subsection{CANAIS ATIVOS UTILIZANDO ERLANG}

Para $N$ canais e um tráfego $\boldsymbol{A}$ a distribuição de Erlang é dada por

$$
p_{n}=\frac{A^{n} / n !}{\sum_{i=0}^{N} A^{i} / i !}
$$


Para o caso de duas células a probabilidade de $k$ assinantes em condições de soft handoff é dada por

$$
p_{k}=\sum_{n=k}^{N}\left(\begin{array}{l}
n \\
k
\end{array}\right) \gamma^{k}(1-\gamma)^{n-k}\left(\frac{A^{n} / n !}{\sum_{i=1}^{N} A^{\prime} / i !}\right)
$$

Para o caso de três células a probabilidade de $k_{l}$ assinantes ativos na região de sobreposição entre duas células e $\boldsymbol{k}_{2}$ assinantes ativos na região de sobreposição entre três células é dado por

$$
\begin{aligned}
p_{k_{1}, k_{2}}=\sum_{n=k_{1}+k_{2}}^{N} & \frac{n !}{k_{1} ! k_{2} !\left(n-k_{1}-k_{2}\right) !}\left(\frac{\gamma-\gamma^{2}}{2}\right)^{k_{1}} \\
& \times \gamma^{2 k_{2}}\left(\frac{2-\gamma-\gamma^{2}}{2}\right)^{n-k_{1}-k_{2}} \frac{A^{n} / n !}{\sum_{i=11}^{N} A^{i} / i !}
\end{aligned}
$$

Observar o aumento da complexidade da expressão em função do acréscimo de regiões de sobreposição.

\subsection{CANAIS ATIVOS UTILIZANDO BERNOULLI}

Supondo que os canais tenham uma atividade $p$ a probabilidade de se terem $n$ canais ativos em $N$ é dada pela distribuição de Bernoulli, tal que:

$$
p_{n}=\left(\begin{array}{l}
N \\
n
\end{array}\right) p^{n \prime}(1-p)^{N-n}
$$

Com a hipótese de canais equiprováveis a atividade $\boldsymbol{p}$ pode ser determinada como a razão entre o tráfego escoado e o número de canais, ou seja

$$
p=\frac{A(1-B)}{N}
$$

onde $B$ é a probabilidade de bloqueio. Para duas células a distribuição $p_{k}$ é então dada por

$$
p_{k}=\sum_{n=k}^{N}\left(\begin{array}{l}
n \\
k
\end{array}\right) \gamma^{k}(1-\gamma)^{n-k}\left(\begin{array}{l}
N \\
n
\end{array}\right) p^{n}(1-p)^{k-n}
$$

que pode ser manipulada para se chegar a

$$
p_{k}=\left(\begin{array}{l}
N \\
k
\end{array}\right)\left(p_{\gamma}\right)^{k}\left(1-p_{\gamma}\right)^{N-k}
$$

onde $p_{\gamma}=\not p$.

Note que (18) é bem mais simples que (13) e (17). Resta verificar se ambas fornecem resultados semelhantes para os objetivos de projeto.

Para três células utilizando Bernoulli para representar o comportamento de tráfego na célula chega-se a

$$
\begin{aligned}
p_{k_{1} \cdot k_{2}=}= & \sum_{n=k_{1}+k_{2}}^{N} \frac{n !}{k_{1} ! k_{2} !\left(n-k_{1}-k_{2}\right) !}\left(\frac{\gamma-\gamma^{2}}{2}\right)^{k_{1}} \gamma^{2 k_{2}} \\
& \times\left(\frac{2-\gamma-\gamma^{2}}{2}\right)^{n-k_{1}-k_{2}}\left(\begin{array}{l}
N \\
n
\end{array}\right) p^{n}(1-p)^{N-n}
\end{aligned}
$$

Manipulando-se esta expressão obtém-se

$$
\begin{aligned}
p_{k_{1}, k_{2}} & =\frac{n !}{k_{1} ! k_{2} !\left(n-k_{1}-k_{2}\right) !}\left[\frac{\gamma-\gamma^{2}}{2} \frac{A(1-B)}{N}\right]^{k_{1}} \\
& \times\left[\gamma^{2} \frac{A(1-B)}{N}\right]^{k_{2}}\left[1-\frac{A}{2 N}(1-B)\left(\gamma+\gamma^{2}\right)\right]^{n-k_{1}-k_{2}}
\end{aligned}
$$

A expressão (20) apresenta uma complexidade computacional bem menor que (14) e (19), possibilitando uma otimização no tempo de cálculo.

\subsection{COMPARAÇÃO ENTRE AS DUAS ABORDAGENS}

Como visto nos itens anteriores, o uso da distribuição de Bernoulli simplifica de forma substancial a equação para o cálculo da distribuição de assinantes em condiçōes de soft handoff. Resta portanto. verificar a proximidade dos resultados utilizando as duas abordagens. Suponha $N=18$ (um número largamente utilizado em projetos CDMA) e $\boldsymbol{B}$ $=2 \%$ e portanto $\boldsymbol{A}=11,5$ erlang. A Figura 3 mostra as curvas de $p_{k}$, para $k=0,2,4,6,8$.

Note na Figura 3 que, para valores práticos de $\gamma(0 \leq \gamma \leq$ $0,5)$, ambas as abordagens fornecem resultados muito próximos entre si.

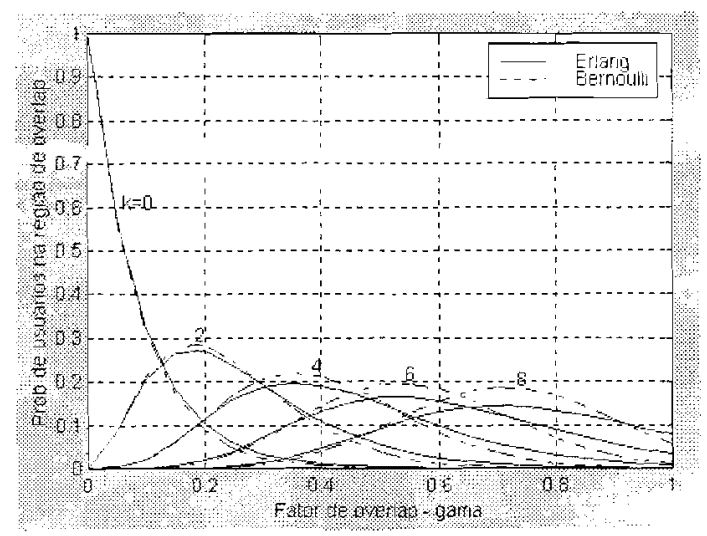

Figura 3. Comparação Erlang versus Bernoulli - duas células.

Para três células esta verificação é mais complexa pois é necessário fixar o número de um dos valores, $\boldsymbol{k}_{\boldsymbol{l}}$ ou $\boldsymbol{k}_{2}$. A Figura 4 apresenta os resultados para $\boldsymbol{k}_{2}=0$ e $\boldsymbol{k}_{1}=0,1$ e 2 .

Pela Figura 4 é possível verificar que a utilização de Bernoulli leva a resultados bastante próximos daqueles obtidos por Erlang. 


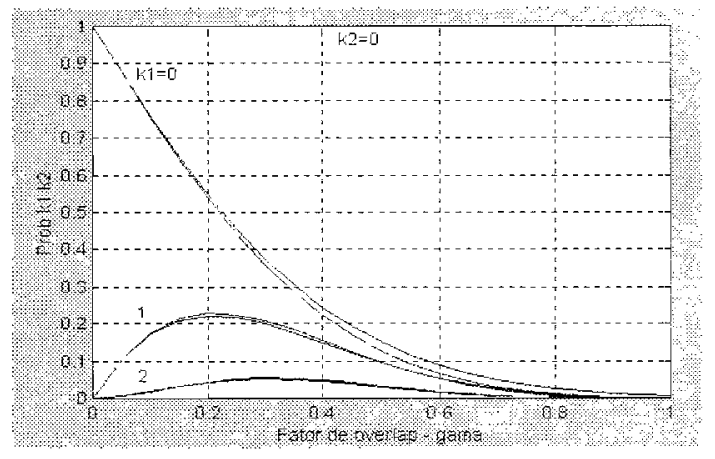

Figura 4. Comparação Erlang versus Bernoulli - três células.

\section{PROBABILIDADE DE BLOQUEIO DE SOFT HANDOFF}

O bloqueio de soft handoff diz respeito à chance de se estar em condição de soft handoff e não se encontrar canais disponíveis na outra célula para a realização do handoff. Para determinar esta probabilidade no caso de duas células devem-se considerar as condições onde não irão existir canais disponíveis na célula alvo para cursar o tráfego de soft handoff gerado pela outra célula. O bloqueio sofrido portanto é devido ao tráfego de soft handoff não cursado. Para duas células esta condição é calculada por

$$
p_{B H O}=\sum_{l=1}^{N_{T}} \sum_{k=l}^{N_{T}} p_{k} p_{n=N_{I}-l+1}
$$

Esta expressão considera as condições onde não existirão sanais disponíveis na célula alvo para cursar o tráfego de soft handoff.

Para o arranjo de três células a célula alvo estará com bloqueio de soft handoff quando o tráfego gerado pelas duas outras células não possa ser cursado por falta de canais na célula alvo. Esta é a condição em que dada uma célula alvo com um número total de canais $N_{T}$, onde $N_{T}$ é a soma dos canais designados para tráfego interno, $N$, mais canais designados para atender tráfego de soft handoff, $N_{H O}$. Para três células na foi obtida uma expressão para cálculo direto do bloqueio de soft handoff. O cálculo desta probabilidade é dada por:

$$
\operatorname{prob}=\sum_{q=1}^{N_{T}} \sum_{k_{1}^{1}=0}^{N_{I}} \sum_{k_{1}^{1}=0}^{N_{F}-k_{1}^{1}} \sum_{k_{1}^{2}=0}^{N_{I}} \sum_{k_{2}^{2}=0}^{N_{T}-k_{1}^{2}} p_{k_{1}^{1} \cdot k_{2}^{1}} p_{k_{1}^{2} \cdot k_{2}^{2}} p_{n=N_{I}-q+1}
$$

onde $k_{x}^{y}$ indica célula $y$ e região de sobreposição $x$. Em (22)

com

$$
k_{1}^{1}+k_{2}^{1}+k_{1}^{2}+k_{2}^{2}+n \leq N_{T}
$$

$$
0 \leq k_{1}^{1} \leq N_{T} \quad e \quad 0 \leq k_{2}^{1} \leq N_{\tau}-k_{1}^{1}
$$

e

$$
0 \leq k_{1}^{2} \leq N_{T} \quad \text { e } \quad 0 \leq k_{2}^{2} \leq N_{T}-k_{1}^{2}
$$

Com estas condições calcula-se a probabilidade de bloqueio de soft handoff. Observar que a probabilidade de canais ativos nas células vizinhas ou na célula alvo pode ser determinada utilizando-se a distribuição de Erlang ou Bernoulli. No trabalho os resultados foram obtidos utilizando somente a distribuição de Erlang para descrever a atividade de canais na célula alvo. Já para atividade de canais nas célula vizinhas foram utilizadas as duas distribuições.

\section{PROBABILIDADE DE OUTAGE}

Ao se adicionarem canais aos canais nominais para fins de -atendimento do tráfego de soft handoff. a ocupação destes canais pode levar a um aumento de interferência. A probabilidade de outage diz respeito à chance de o número de canais nominais serem excedidos. Seja $N_{T}$ o número total de canais tal que $N_{T}-N$ corresponde ao número de canais acrescentados aos nominais. Supondo que as chamadas em condições de soft handoff permaneçam ativas mesmo na ausência de canais livres na outra célula, a probabilidade de outage para duas células é dada por:

$$
p_{\text {omt }}=\sum_{i=n}^{N_{t}} \sum_{k=1}^{N_{T}} p_{k} p_{n=N_{T}-1}+\sum_{q=N+1}^{N_{f}-1} \sum_{k=1}^{q} p_{k} p_{n=q-k}
$$

Para o caso de três células a probabilidade de outage é determinada por:

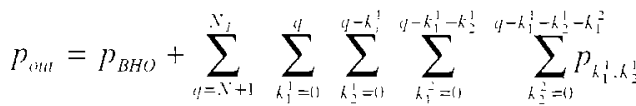

$$
\begin{aligned}
& \times p_{k_{1}^{\prime}, k i} p_{n=t j-k_{1}^{1}-k_{2} !-k_{j}-k}
\end{aligned}
$$

Observar que foi utilizada a probabilidade de bloqueio de soft handoff para determinação da probabil.i...; de outage.

\section{ESTRATÉGIAS DE ALOCAÇÃO DE CANAIS}

O handoff é uma operação importante em qualquer sistema celular. Para o sistema CDMA esta operação ganha importância ainda maior em função de o usuário utilizar recursos do sistema, em excesso a um mínimo necessário. Este fato afeta fortemente o dimensionamento do sistema que deverá suportar esta operação e atender aos requisitos de qualidade de serviço. Importante notar que mais crítico que bloqueio na originação de uma chamada é a queda de chamada em curso por falta de canal na operação de soft handoff. A contribuição do trabalho é no sentido de definir novos parâmetros que devem ser levados em consideração no planejamento de sistemas celulares CDMA

Trabalha-se primordialmente com a questão de interferência, que em sistemas CDMA leva a uma degradação suave à medida que vai-se aumentando o número de usuários no sistema. Utilizam-se aqui os conceitos de tráfego aparente e tráfego real, já apresentados 
anteriormente. A determinação do tráfego real na metodologia proposta será conseqüência tanto do bloqueio de soft handoff quanto da interferência. Para o dimensionamento de canais para satisfazer a condição de soft handoff utiliza-se um número nominal de canais $N$ com um tráfego $\boldsymbol{A}$ para um bloqueio $\boldsymbol{B}$. Este tráfego é denominado de aparente. A carga aparente é obtida pela fórmula de Erlang, para um dado grau de serviço e um número de canais para o qual o nível de interferência encontra-se em patamares aceitáveis. Aqui. somente será considerado que existe um número nominal de canais $N$, que caso todos os canais estejam ativos a interferência adicional produzida pode ser desconsiderada, permitindo o funcionamento adequado do sistema. Para valores maiores que $N$ não significa que o sistema deixe de operar e sim uma condição de interferência que degrade suavemente a comunicação com o aumento de $N$. Resta portanto verificar as probabilidades envolvidas nesta condição. Trabalha-se, assim. com as probabilidades de existir interferência e a probabilidade de ocorrer bloqueio de soft handoff. A questão, neste caso, é determinar a probabilidade da ocorrência dos eventos quando é excedido este número de canais $N$. A título de exemplo esta condição nominal considera um tráfego $A$ de 11,5 Erlangs e uma probabilidade de bloqueio $\boldsymbol{B}$ de $2 \%$. Utilizando a tabela de Erlang-B chega-se a $N=18$ canais.

Nas estratégias de planejamento propostas, para uma proporção $\gamma$ de sobreposição entre células, adicionam-se ou subtraem-se canais aos $N$ canais nominais para atender aos requisitos de probabilidade de bloqueio de soft handoff e de outage. Note que ao se acrescentar canais aos canais nominais a probabilidade de bloqueio de soft handoff deverá diminuir. Por outro lado, a probabilidade de interferência deverá aumentar, ou seja, a probabilidade de outage deverá aumentar. No outro extremo, apenas subtraindo-se canais dos canais nominais, técnica aplicada comumente, a interferência não ultrapassará aquela obtida com o uso de canais nominais, mas a capacidade final do sistema diminuirá para se atingir a probabilidade de bloqueio de soft handoff desejada. Um compromisso entre se adicionar e se subtrair uma quantidade adequada de canais em torno dos canais nominais poderá ser atingida, para se atender aos critérios de projeto de sistema no que diz respeito à questão da interferência e do bloqueio de soft handoff. A estratégia de planejamento proposta se presta a este objetivo introduzindo a definição de novos parâmetros a serem considerados, ou seja. a probabilidade de bloqueio de soft handoffe a probabilidade de outage.

Trabalha-se com uma célula alvo onde serão analisadas as probabilidades de bloqueio de soft handoff e outage, sendo que as outras células vizinhas funcionam como geradoras de tráfego de soft handoff nas regiões de sobreposição. Para tal fim, serão utilizadas as distribuições de Erlang e Bernoulli para o tráfego nas células vizinhas geradoras de tráfego de soft handoff. Para os propósitos destas técnica $N$ é o número nominal de canais para o qual a interferência pode ser desprezada. $N_{T}$ é o número total de canais alocados na célula e $N_{I N T}$ o número de canais para atendimento do tráfego interno na célula alvo.

\subsection{ESTRATÉGIA DE AUMENTO DE CANAIS}

Nesta estratégia de alocação parte-se do número nominal de canais $N$ e são acrescidos canais chegando-se a $N_{T}$ canais alocados na célula alvo. Deve-se agora analisar as consequiências deste aumento do número de canais alocados na célula relativamente à probabilidade de bloqueio de soft handoff e probabilidade de outage. Notar que neste tipo de estratégia o número de canais internos se confunde com o número nominal de canais $N$, isto é $N_{I N T}=N$.

Parte-se. nesta estratégia, para o aumento do número de canais alocados na célula alvo no sentido de atender ao tráfego de soft handoff. $\mathrm{O}$ aumento do número de canais implica em diminuição da probabilidade bloqueio de soft handoff. Porém, com o aumento do número de canais alocados na célula existirá a probabilidade de canais ativos em excesso causando interferência. Esta probabilidade de ocorrer interferência será avaliada pela probabilidade de outage. A probabilidade de bloqueio na célula permanece a mesma uma vez que não é alterado o número nominal de canais $N$ para atender ao tráfego na célula. e conseqüentemente o tráfego aparente permanece constante. Esta técnica de alocação se aplicaria nos casos onde existe a necessidade de diminuição da probabilidade de bloqueio de soft handoff.

A Figura 5 ilustra esta estratégia. Ou seja, parte-se de um valor nominal de canais $N$, e são acrescidos canais chegando-se a $N_{T}$ canais alocados na célula. Na Figura 5 também está apresentada a expectativa quanto às probabilidades de bloqueio, bloqueio de soft handoff e outage.

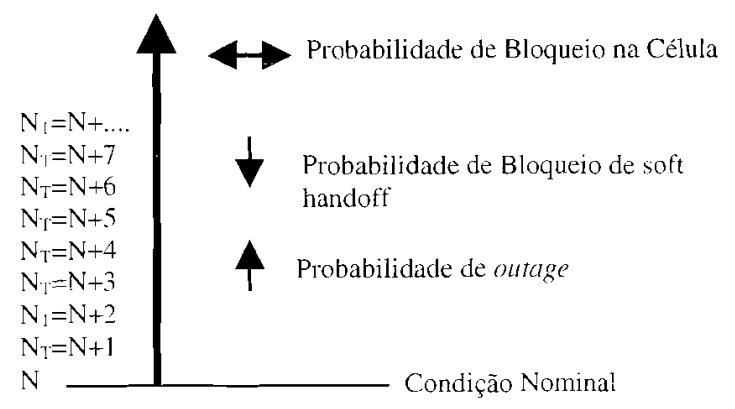

Figura 5. Estratégia de aumento de canais.

A estratégia apresentada na Figura 5 será utilizada para arranjos de duas e três células. Serão avaliadas as probabilidades de bloqueio de soft handoff e outage $\mathrm{cm}$ função da proporção de sobreposição $\gamma$ e do número total de canais $N_{T}$ alocados na célula.

\subsection{ESTRATÉGIA DE DIMINUIÇÃO DE CANAIS}

Neste tipo de estratégia parte-se novamente da condição nominal $\operatorname{com} N$ canais alocados na célula alvo. Porém, neste caso o valor nominal de canais é mantido como o valor máximo e considerado como sendo $N_{T}$, ou seja, o valor total de canais alocados na célula alvo. Deste valor são 
subtraídos canais, que se prestam para atendimento do tráfego de soft handoff.

Portanto tem-se para esta técnica $N_{T}=N$. Com a diminuição do número de canais alocados haverá uma conseqüente diminuição do tráfego aparente. Assim, teremos um número de canais $N_{I N^{\prime} T}$ para cursar o tráfego da célula. A diferença de canais $N_{T}-N_{I N T}$ será destinada para cursar o tráfego de soft handoff. Variando-se a proporção $\gamma$ de sobreposição entre células avalia-se a probabilidade de bloqueio de soft handoff. Observar que nesta estratégia não se ultrapassa o número nominal de canais, pois $N_{T}=N$. Sendo assim, não ocorrerá o caso de interferência, sendo esta desprezada. Portanto, a probabilidade de bloqueio de soft handoff será a única a ser avaliada, pois a probabilidade de outage não se aplica uma vez que não é ultrapassada a condição nominal.

Na Figura 6 estão apresentadas as condições a serem analisadas nos arranjos de 2 e 3 células.

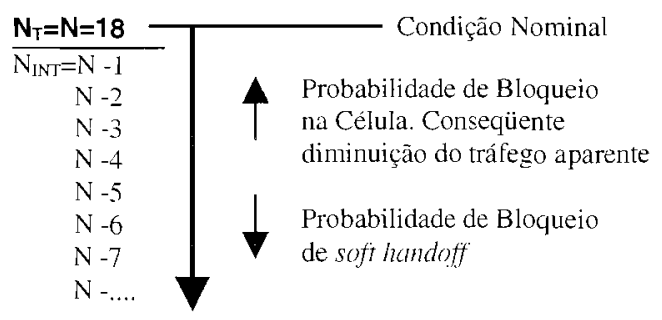

Figura 6. Estratégia de diminuição de canais.

Espera-se neste caso uma diminuição da probabilidade de bloqueio de soft handoff e um conseqüente aumento na probabilidade de bloqueio na célula, provocando uma diminuição no tráfego aparente na célula para manutenção do bloqueio em 2\%. Esta situação se contrasta com a técnica de alocação apresentada do item anterior, pois naquele caso o número de canais para cursar o tráfego aparente é mantido constante. Esta técnica apresentada na Figura 6 é utilizada comumente no planejamento de sistemas celulares CDMA.

\subsection{ESTRATÉGIA DE AUMENTO E DIMINUIÇÃO DE CANAIS}

Nas duas técnicas de alocação apresentadas anteriormente foram utilizadas estratégicas opostas com características bastante diferentes. Aqui é apresentada uma estratégia que utiliza os dois princípios, onde são acrescidos canais aos $N$ nominais para cursar o tráfego de soft handoff e também é feita a subtração de canais em relação aos nominais para cursar o tráfego na célula. Neste tipo de alocação parte-se também da condição nominal com $N$ canais.

A diminuição do número de canais para cursar o tráfego da célula ocasiona uma diminuição do tráfego aparente uma vez que existirão menos canais para cursar o tráfego da célula. Por outro lado, existe o aumento do número de canais para cursar o tráfego de soft handoff. A estratégia proposta segue portanto um processo onde são somados canais aos nominais e verificada a condição de probabilidade de bloqueio de soft handoff e probabilidade de outage quando da diminuição de canais alocados para cursar o tráfego interno na célula alvo. A Figura 7 mostra este tipo de estratégia de alocação.

A cada etapa de adição de canais aos nominais é feita a avaliação das probabilidades envolvidas diminuindo-se assim os canais alocados para cursar o tráfego interno. O processo é repetido até obter-se uma condição satisfatória tanto com relação ao bloqueio de soft handoff quanto da probabilidade de outage.

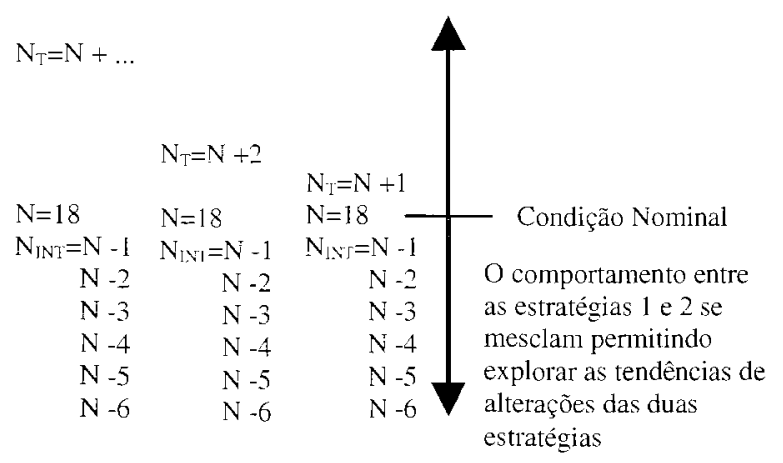

Figura 7. Estratégia de diminuição e aumento de canais.

\section{RESULTADOS}

Neste item são apresentados os resultados das estratégias de alocação para duas e três células. Aqui também foi utilizado os valores apresentados no item 6 . Os resultados são obtidos em função da proporção de sobreposição entre as células.

\subsection{RESULTADOS PARA DUAS CÉLULAS}

Utilizando a primeira estratégia, onde admite-se a condição de apenas se acrescentarem canais aos canais nominais. os resultados são aqueles mostrados pela Figura 8, para a probabilidade de bloqueio de soft handoff, e pela Figura $n$ para a probabilidade de outage.

Nesta condição variou-se o número de canais $N_{T}$ de $2 u$. 24. Note que a probabilidade de outage é praticamente a mesma para os diversos valores de $N_{T}$. A probabilidade de bloqueio de soft handoff, no entanto, cai substancialmente à medida em que se acrescentam canais aos canais nominais.

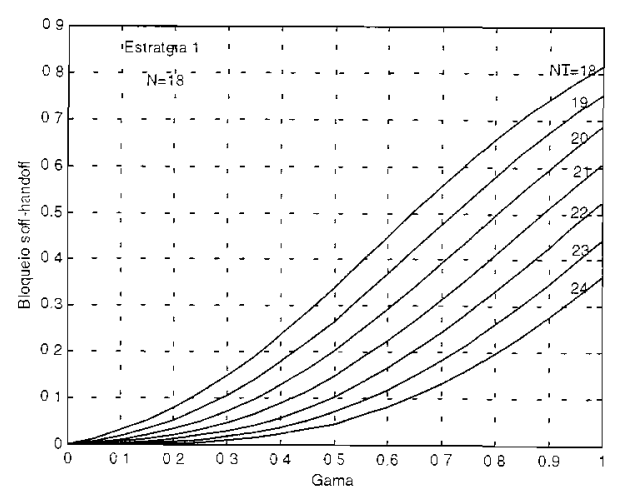

Figura 8. Bloqueio de soft handoff-duas células - aumento de canais. 


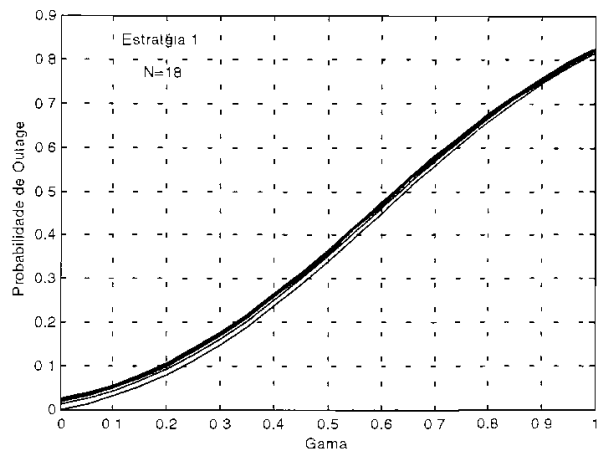

Figura 9. Probabilidade de outage - duas células - aumento de canais.

A Figura 10 apresenta uma comparação entre a utilização de distribuição de Erlang e Bernoulli na obtenção da probabilidade de bloqueio de soft handoff. Observar a grande proximidade das curvas, principalmente para valores de $\gamma$ abaixo de $50 \%$

Utiliza-se agora a segunda estratégia, onde somente se diminui o número de canais em relação ao número nominal de canais. A Figura 11 apresenta a probabilidade de bloqueio de soft handoff. Para esta condição somente define-se probabilidade de bloqueio de soft handoff uma vez que não se ultrapassa o número nominal de canais.

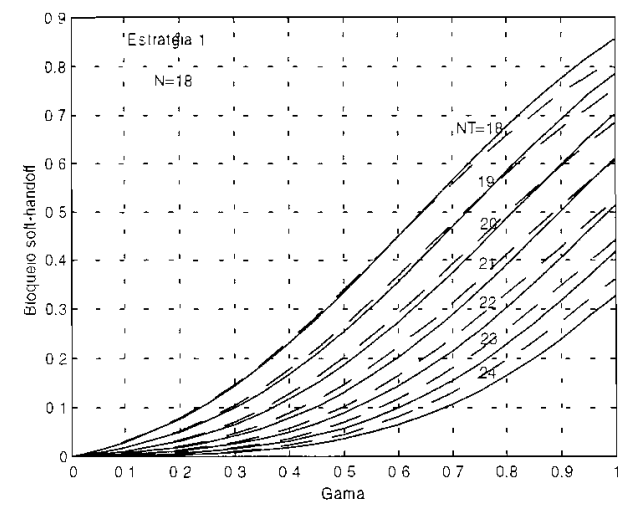

Figura 10. Comparação Erlang e Bernoulli duas células.

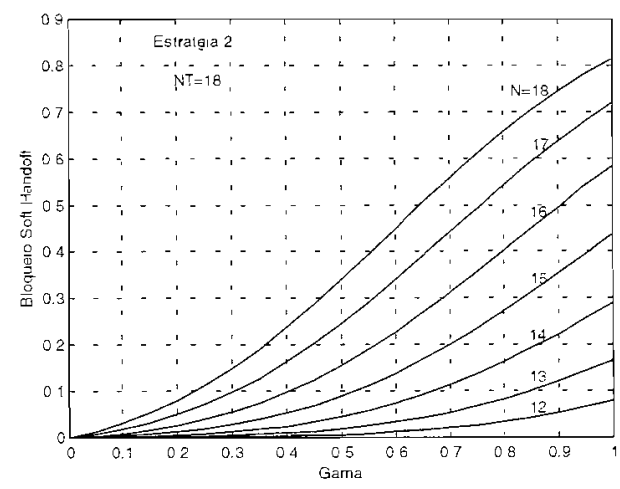

Figura 11. Bloqueio de soft handoff - duas células - diminuição de canais.
Nesta condição variou-se o número de canais $N$ de 18 a 12. Como era de se esperar o bloqueio de soft handoff cai substancialmente. Porém, também cai o tráfego aparente.

Utiliza-se agora a terceira estratégia, onde utilização uma mescla das duas estratégias anteriores. Para demonstração da metodologia será apresentado os resultados para $N_{T}=21$. A Figura 12 apresenta a probabilidade de bloqueio de soft handoff e a Figura 13 apresenta a probabilidade de outage. Observar a possibilidade de se trabalhar com a variação do número de canais em função de $\gamma$ atendendo a requisitos de projeto.

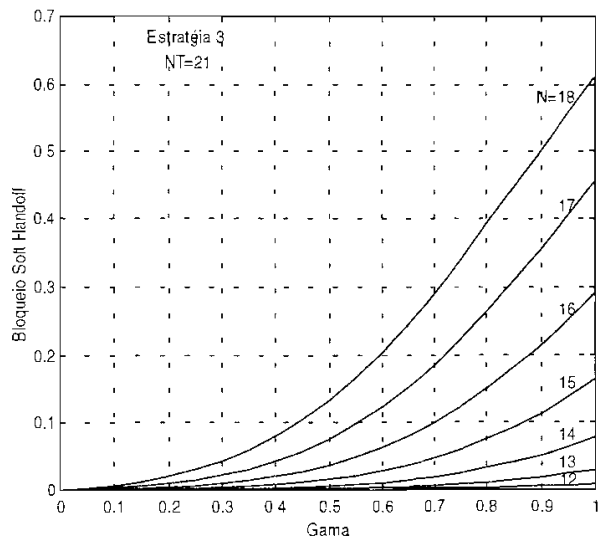

Figura 12. Bloqueio de soft handoff - duas células - aumento e diminuição de canais.

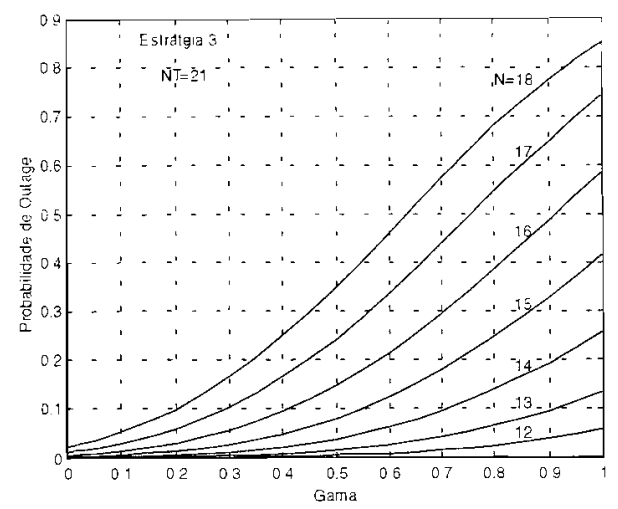

Figura 13. Probabilidade de outage duas células aumento e diminuição de canais

\subsection{RESULTADOS PARA TRÊS CÉLULAS}

Utilizando a primeira estratégia onde admite-se a condição de apenas se acrescentarem canais aos canais nominais. os resultados são aqueles mostrados pela Figura 14. para a probabilidade de bloqueio de soft handoff, e pela Figura 15 para a probabilidade de outage.

Nesta condição variou-se o número de canais $N_{T}$ de 20 a 24. Note que a probabilidade de outage é praticamente a mesma para os diversos valores de $N_{T}$. A probabilidade de bloqueio de soft handoff, no entanto, cai substancialmente à medida em que se acrescentam canais aos canais nominais. 


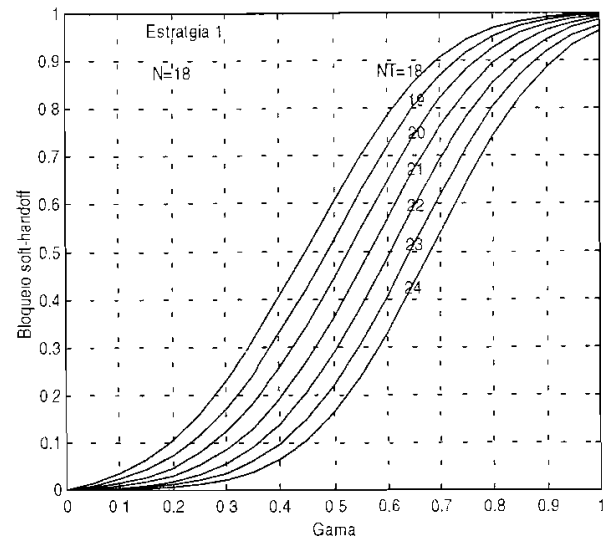

Figura 14. Bloqueio de soft handoff - três células - aumento de canais.

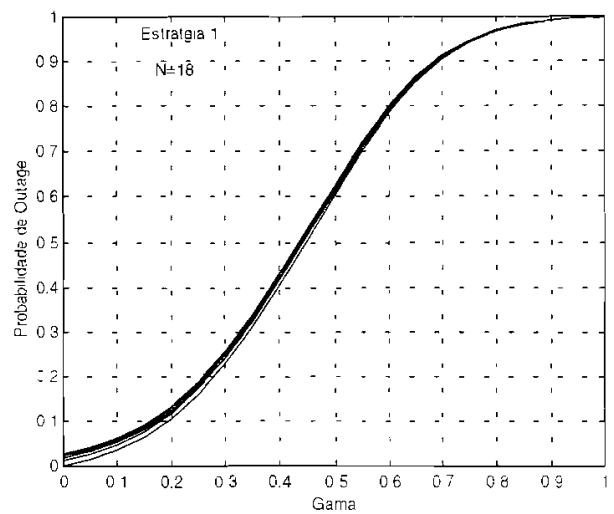

Figura 15. Probabilidade de outage - três células - aumento de canais.

Utiliza-se agora a segunda estratégia, onde somente se diminui o número de canais em relação ao número nominal de canais.

A Figura 16 apresenta a probabilidade de bloqueio de soft handoff. Para esta condição somente define-se probabilidade de bloqueio de soft handoff uma vez que não se ultrapassa o número nominal de canais.

Nesta condição, variou-se o número de canais $N$ de 18 a 12. Como era de se esperar o bloqueio de soft handoff cai substancialmente. Porém, também cai o tráfego aparente.

Utiliza-se agora a terceira estratégia. onde utilização uma mescla das duas estratégias anteriores. Para demonstração da metodologia será apresentado os resultados para $N_{T}=22$. A Figura 17 apresenta a probabilidade de bloqueio de soft handoffe a Figura 18 apresenta a probabilidade de outage.

Os resultados apresentados neste item são utilizados no próximo item na aplicação da metodologia proposta.

\section{APLICAÇÃO DA METODOLOGIA}

Para efeito de demonstração do método proposto somente será utilizado o arranjo de três células. A não utilização dos resultados para duas células se justifica por

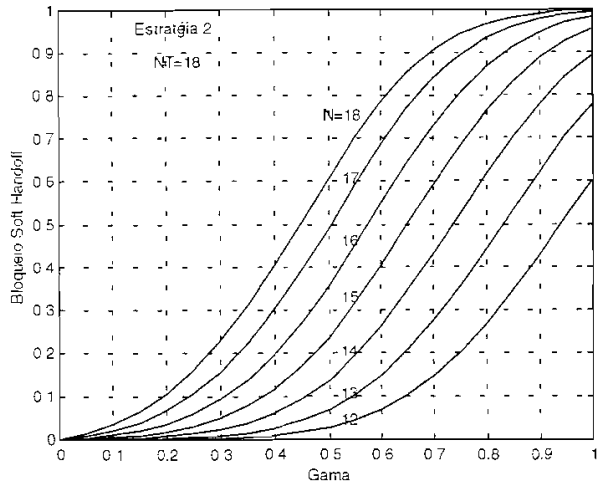

Figura 16. Bloqueio de Soft handoff - três células - diminuição de canais.

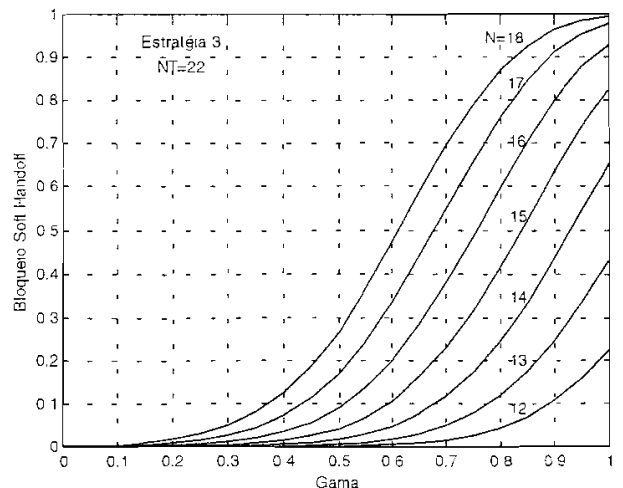

Figura 17. Bloqueio de soft handoff - três células - aumento e diminuição de canais.

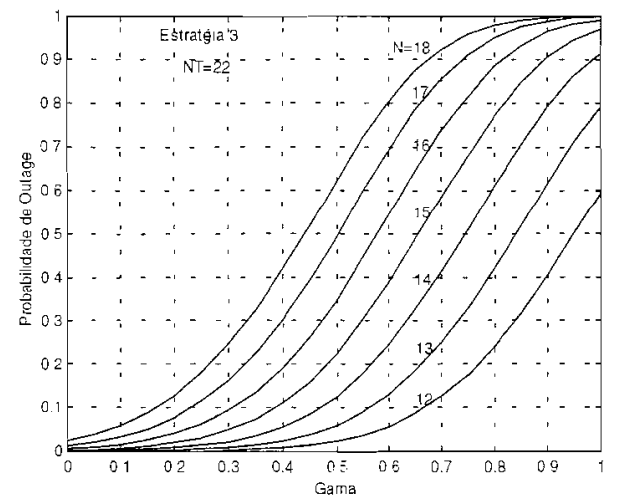

Figura 18. Probabilidade de outage - três células - aumento e diminuição de canais.

sua limitada aplicação, servindo esta abordagem como consolidação dos resultados obtidos no arranjo de três células. Será verificado o desempenho das três estratégias de planejamento apresentadas no item 6. Observar que, de fato, o método proposto neste trabalho é uma mescla da primeira estratégia de alocação. Figura 5, com a segunda 
estratégia de alocação, Figura 6, resultando na terceira estratégia de alocação apresentada na Figura 7. Sendo assim, as duas primeiras estratégias servem de base para a análise a ser realizada considerando as probabilidades de bloqueio de soft handoff e outage.

Para o exercício de planejamento será utilizado o valor de $2 \%$ para a probabilidade de bloqueio de soft handoff. Ou seja, é permitido um bloqueio de até $2 \%$. Este valor deverá ser um dado de entrada no dimensionamento do sistema, sendo um valor a ser atendido no planejamento.

Será utilizado o valor de $8 \%$ para a probabilidade de outage, definido arbitrariamente, e serve somente como referência para demonstração da técnica de planejamento apresentada. Este também deve ser um valor de entrada no dimensionamento do sistema, sendo um valor a ser atendido no planejamento.

Admitindo-se a primeira estratégia de alocação de apenas acrescentar-se canais aos canais nominais para atendimento do soft handoff haverá, conseqüentemente uma diminuição da probabilidade de bloqueio de soft handoff. A Figura 14 apresenta o comportamento da probabilidade de bloqueio de soft handoff em função da proporção de sobreposição entre células, onde são incluídos de 1 a 6 canais ao 18 canais nominais totalizando de 19 a 24 canais alocados na célula. Observar que para $\gamma=40 \%$ a probabilidade de bloqueio de soft handoff é de $7 \%$ para 24 canais alocados na célula, não atendendo aos $2 \%$ estabelecidos como meta. Para este número de canais alocados na célula a probabilidade de outage ficará por volta de $44 \%$ para 24 canais, como mostra a Figura 15 . Este valor é bastante acima dos $8 \%$ especificados.

Utiliza-se agora a segunda estratégia, onde são subtraídos canais dos canais nominais. Utilizando a Figura 16 chega-se para $\gamma$ de $40 \%$ e $N=12$ a um bloqueio de soft handoff de 1\%. Porém, apenas subtraindo-se canais dos canais nominais a interferência não ultrapassará àquela obtida com o uso de canais nominais, sendo definida como $0 \%$ para este caso, ficando portanto muito abaixo dos $8 \%$ especificados. Outro ponto a ser analisado é a capacidade de tráfego real do sistema que diminuirá para se atingir a probabilidade de bloqueio de soft handoff desejada, passando dos 11.5 Erlangs, que é o tráfego aparente. para um trálego real de 6,61 Erlangs. Esta não é uma situação razoável por privilegiar o tráfego de soft handoff em detrimento da capacidade do sistema.

Um compromisso entre se adicionar e se subtrair uma quantidade adequada de canais em torno dos canais nominais poderá ser atingida para se atender aos critérios de projeto de sistema, no que diz respeito à questão da interferência e de bloqueio de soft handoff. Parte-se portanto para o exercício de planejamento onde são adicionados canais aos nominais e subtraídos canais para liberar canais para tráfego de soft handoff. Para um total de 22 canais verifica-se na Figura 17 que são necessários 7 canais para soft handoff para que a probabilidade de bloqueio de soft handoff fique por volta de $2 \%$, restando portanto 15 canais para cursar o tráfego interno. Pela Figura 18 observa-se porém que a probabilidade de outage fica acima dos $8 \%$ especificados. Utilizando-se porém 8 canais para cursar o tráfego de soft handoff e 14 canais para o tráfego interno da célula chega-se a uma probabilidade de bloqueio de soft handoff inferior a $2 \%$ e uma probabilidade de outage por volta de $8 \%$.

Esta última condição parece bastante razoável pois comparando-se com a segunda estratégia onde o tráfego real era de 6,61 Erlangs obtêm-se um trátego real de 8,2 Erlangs, atendendo-se aos requisitos de planejamento estabelecidos para bloqueio de soft handoff e outage.

\section{CONCLUSÕES}

Este artigo apresentou uma nova metodologia de dimensionamento de canais de soft handoff em sistemas CDMA. Nesta metodologia são consideradas tanto a probabilidade de outage quanto a probabilidade de bloqueio de soft handoff na determinação do número de canais da célula. Em contraste com as metodologias existentes, esta proposta é simples, de fácil aplicação e pode ser utilizada para distribuições diversas de tráfego.

\section{REFERÊNCIAS}

[1] Branquinho. Omar C., Yacoub M. D., Dimensionamento de Canais de Soft Handoffem Sistemas CDMA, SBT - 1999. Vila Velha ES.

[2] Branquinho. Omar C.. Yacout M. D.. Dimensionamento de Canais de Soft Handoffem Sistemas CDMA. SBT - 1999. Vila Velha ES.

[3] Viterbi. Adrew J. Principles of Spread Spectrum Conmunication, Addison-Wesley Publishing Company. 1995.

[4] Yang. Samuel C.. CDMA RF System Engineering, Artech House, 1998.

[5] Guedes, L. G. R., Yacoub M. D., Sobreposição entre células com restrição de cobertura, Journal of Microwaves Optoelectronics, vol. 1. n"1. pp. 10-29. Maio 1997.

[6] ---, Boundaries between cells in Nakagami fading conditions, SBT/IEEE International Telecommunications Symposimm, ITS94. Rio de Janeiro, August 94.

[7] Papoulis, Athanasios. Probability \& Statistics, Englewood Cliffs : Prentice Hall. 1990. 454 p.

Omar Carvalho Branquinho graduou-se pelo INATEL em 1985 e mestrado pela UNICAMP em 1991. Está finalizando doutoramento pela UNICAMP. É pesquisador da Fundação Centro de Pesquisa - CPqD - desde 1985, trabalhando em comunicaçōes celulares desde 1989. Vem participando na especificação dos sistemas móveis avançados na ITU. Professor da Universidade São Francisco deste 1995.

Michel Daoud Yacoub graduou-se pela Universidade de Campinas em 1978. Obteve o títulos de Mestre em Engenharia Elétrica pela mesma Universidade, em 1983 e o de Ph.D. pela University of Essex, em 1988. Atua no Departamento de Comunicações da Faculdade de Engenharia Elétrica e de Computaçã̃o da Unicamp desde 1989, onde ocupa o cargo de Professor Titular. 